\title{
Evaluation by exercise testing and atrial pacing of results of aorto-coronary bypass surgery
}

\author{
R. Balcon, M. Honey, A. F. Rickards, ${ }^{1}$ M. F. Sturridge, W. Walsh, ${ }^{2}$ R. K. Wilkinson, ${ }^{3}$ and \\ J. E. C. Wright
}

From the London Chest Hospital and Cardiothoracic Institute

The results are reported of investigation and surgery in rog patients with angina pectoris who have undergone aorto-coronary bypass surgery. There were 7 hospital and no late deaths. Of 50 patients followed for more than 6 months, 36 became symptom free and a further 12 improved. This clinical improvement was accompanied by significant increase in exercise performance which was related to the preoperative state of the left ventricle. Thirty-three patients were catheterized postoperatively and in this group also clinical improvement correlated well with the results of exercise and pacing tests. Of 52 grafts, $43(83 \%)$ were patent and there was a close association between evidence of revascularization and clinical result.

Aorto-coronary bypass surgery is now widely used in the treatment of patients with coronary artery disease. Previous operations have been abandoned largely because of the lack of objective evidence of both symptomatic improvement and revascularization. In the assessment of this operation we have therefore used exercise ergometry and cardiac pacing to provide a more objective assessment of the value of this procedure.

\section{Patients and methods}

Patients presenting with angina pectoris from November I970 to June I973 were considered for surgery and admitted to hospital for evaluation. Digoxin and betaadrenergic blocking agents were stopped 48 hours before admission. Angina was graded from I to 4 using the same criteria as the New York Heart Association grading for dyspnoea. Routine chest radiographs and electrocardiograms were obtained, and fasting serum cholesterol was estimated. The patients performed a standardized exercise test in the upright position on a bicycle ergometer with recording of a bipolar chest lead. The exercise started at $150 \mathrm{kpm} / \mathrm{min}$ and increased by increments of $150 \mathrm{kpm} / \mathrm{min}$ every 2 minutes until the

Received 4 March 1974.

1 Present address: National Heart Hospital, London W.r.

2 Present address: Department of Cardiology, University of Chicago, Chicago, Illinois 60637 , U.S.A.

${ }^{3}$ Present address: Chermside Hospital, Chermside 4032, Queensland, Australia. development of angina, $2 \mathrm{~mm}$ ST segment depression on the electrocardiogram, dyspnoea, or fatigue.

Left and right heart cardiac catheterization was performed from the right arm after premedication with 20 to $25 \mathrm{mg}$ of intramuscular diazepam. The resting left ventricular end-diastolic pressure was recorded, and the heart was then paced usually from the right atrium (or right ventricle if block developed) until the patient developed angina or a rate of 180 b.p.m. was reached. Left ventriculography was performed in the right anterior oblique projection and followed by selective coronary angiography in multiple projections, preferably using the Sones technique. A caesium iodide image intensifier was used and $35 \mathrm{~mm}$ cine film was exposed at 36 frames per second.

The angiographic severity of coronary artery disease was represented by a numerical score. Each major vessel was scored on a scale of 0 to 4 ; O was normal, 4 total occlusion, and I to 3 stenoses of increasing severity. A similar system was applied to the left ventriculogram. Grade $O$ was normal and $I$ to 4 increasing extent of dyskinesia.

\section{Surgery}

Operation was usually performed within one month of investigation. After removal of a length of saphenous vein, the coronary anastomoses were performed under normothermic cardiopulmonary bypass with intermittent aortic cross clamping. In some of the later patients endarterectomy was done before graft insertion, and in some direct internal mammary artery anastomosis was used 


\section{Postoperative follow-up}

The patients were seen at 3-monthly intervals for the first year and then at 6-monthly intervals. Exercise tests were performed at each visit. A small group of patients had a postoperative catheterization similar to the preoperative study, but including graft angiography.

\section{Clinical data}

The clinical data are shown in Table $\mathrm{I}$. There were I09 patients, 95 men and 14 women, with ages ranging from 30 to 65 , with an average of 50 years. Seventy patients had severe angina (grades 3 and

TABLE I Clinical details

\begin{tabular}{|c|c|c|c|c|c|c|c|c|c|c|c|}
\hline \multirow{2}{*}{\multicolumn{2}{|c|}{$\begin{array}{l}\text { Case Age } \\
\text { No. (yr) }\end{array}$}} & \multirow[t]{2}{*}{ Sex } & \multicolumn{2}{|c|}{ Angina } & \multirow{2}{*}{$\begin{array}{l}\text { Previous } \\
\text { myo- } \\
\text { cardial } \\
\text { infarct }\end{array}$} & \multirow[t]{2}{*}{$\begin{array}{l}\text { Electro- } \\
\text { cardiogram }\end{array}$} & \multirow[t]{2}{*}{$\begin{array}{l}\text { Chest } \\
\text { radiograph }\end{array}$} & \multirow{2}{*}{$\begin{array}{l}\text { Chole- } \\
\text { sterol } \\
(\mathrm{mg} / \\
\text { IOO } \mathrm{ml})\end{array}$} & \multirow[t]{2}{*}{$\begin{array}{l}\text { Nitro- } \\
\text { glycerin }\end{array}$} & \multirow{2}{*}{\multicolumn{2}{|c|}{$\begin{array}{l}\text { Beta adrenergic } \\
\text { blocking agents } \\
\qquad \begin{array}{l}\text { Dose } \mid \text { day } \\
(\mathrm{mg})\end{array}\end{array}$}} \\
\hline & & & Grade & $\begin{array}{l}\text { Duration } \\
\text { (mth) }\end{array}$ & & & & & & & \\
\hline $\mathbf{I}$ & 53 & $\mathbf{M}$ & 4 & 96 & + & Abnormal & Normal & 540 & + & Propranolol & 160 \\
\hline 2 & 58 & $\mathrm{~F}$ & 3 & 84 & - & Abnormal & Normal & 300 & + & Propranolol & 120 \\
\hline 3 & 57 & $\mathbf{M}$ & 4 & 24 & - & Abnormal & Normal & 260 & + & Propranolol & 160 \\
\hline 4 & 48 & $\mathbf{M}$ & 3 & 24 & - & Normal & Normal & 290 & + & Practolol & 400 \\
\hline 5 & 50 & $\mathbf{M}$ & 3 & 48 & - & Abnormal & Normal & 340 & - & Propranolol & 140 \\
\hline 6 & 57 & $\mathbf{M}$ & 3 & 60 & - & Abnormal & Normal & 240 & + & Propranolol & 140 \\
\hline 7 & 65 & $\mathbf{M}$ & 3 & I9 & - & Abnormal & Normal & 230 & + & Propranolol & 160 \\
\hline 8 & 30 & $\mathbf{F}$ & 2 & 228 & - & Abnormal & Normal & 234 & + & Practolol & 400 \\
\hline 9 & 58 & $\mathbf{M}$ & 3 & 36 & + & Abnormal & Normal & 303 & 一 & Practolol & 300 \\
\hline 10 & 58 & $\mathbf{F}$ & 3 & 12 & - & Normal & Normal & 188 & + & Propranolol & 160 \\
\hline I I & 56 & $\mathbf{F}$ & 3 & 36 & - & Normal & Normal & 268 & + & Oxprenolol & 160 \\
\hline 12 & 56 & $\mathbf{M}$ & 2 & 168 & + & Abnormal & Normal & 250 & + & Propranolol & 320 \\
\hline 13 & 62 & $\mathbf{M}$ & 3 & 24 & - & Abnormal & Enlarged heart & 395 & + & Practolol & 800 \\
\hline 14 & 62 & $\mathbf{M}$ & 4 & 120 & - & Abnormal & Normal & 215 & + & Propranolol & 120 \\
\hline I5 & 46 & $\mathbf{M}$ & 2 & I8 & - & Abnormal & Normal & 247 & + & Propranolol & 160 \\
\hline I6 & 36 & $\mathbf{M}$ & 3 & 14 & - & Abnormal & Normal & 236 & + & Practolol & 600 \\
\hline I7 & 53 & $\mathbf{F}$ & 3 & 24 & - & Abnormal & Normal & 360 & + & Propranolol & 240 \\
\hline 18 & 55 & $M$ & 3 & 48 & + & Abnormal & Enlarged heart & 294 & + & Propranolol & 160 \\
\hline I9 & 57 & $\mathbf{M}$ & 3 & 144 & + & Abnormal & $\begin{array}{l}\text { Enlarged heart } \\
\text { and pulm. } \\
\text { venous }\end{array}$ & & & & \\
\hline & & & & & & & congestion & 302 & + & Practolol & 800 \\
\hline 20 & $6 I$ & $\mathbf{M}$ & 2 & 9 & + & Abnormal & Normal & 202 & + & Practolol & 800 \\
\hline $2 I$ & 55 & $\mathbf{M}$ & 2 & 6 & + & Abnormal & Enlarged heart & 272 & + & Practolol & 400 \\
\hline 22 & 47 & $\mathbf{F}$ & 3 & 12 & - & Normal & Normal & 256 & + & Propranolol & 320 \\
\hline 23 & $5 I$ & $\mathbf{M}$ & 3 & 36 & - & Abnormal & Normal & 282 & + & Propranolol & 160 \\
\hline 24 & 42 & $\mathbf{M}$ & 3 & 32 & + & Abnormal & Normal & 274 & - & Practolol & 300 \\
\hline 25 & 40 & $\mathbf{M}$ & 3 & 60 & + & Abnormal & Enlarged heart & $\star$ & + & None & - \\
\hline 26 & 50 & $\mathbf{F}$ & 3 & ro & - & Abnormal & Normal & 200 & + & Propranolol & 240 \\
\hline 27 & 44 & $\mathbf{M}$ & 3 & 7 & + & Abnormal & Normal & $\star$ & + & Practolol & 800 \\
\hline 28 & 55 & $\mathbf{M}$ & 4 & I44 & - & Abnormal & Normal & 370 & + & Practolol & 600 \\
\hline 29 & 47 & $\mathbf{F}$ & 3 & 9 & - & Abnormal & Normal & 226 & + & Propranolol & 60 \\
\hline 30 & 35 & $M$ & 2 & 14 & - & Abnormal & Normal & 242 & + & Practolol & 600 \\
\hline 31 & 39 & $\mathbf{M}$ & 4 & 6 & - & Normal & Normal & 236 & + & Practolol & 600 \\
\hline 32 & 30 & $\mathbf{M}$ & 3 & 5 & - & Abnormal & Normal & 376 & + & Practolol & 800 \\
\hline 33 & 55 & $\mathbf{M}$ & 3 & 36 & - & Abnormal & Normal & 312 & + & Propranolol & 120 \\
\hline 34 & 49 & $\mathbf{M}$ & 3 & 6 & - & Abnormal & Normal & 280 & + & Propranolol & 120 \\
\hline 35 & 43 & $M$ & 2 & 6 & - & Normal & Normal & $\star$ & + & Practolol & 300 \\
\hline 36 & 54 & $\mathbf{M}$ & 3 & 84 & - & Abnormal & Enlarged heart & 308 & + & Practolol & 400 \\
\hline 37 & 50 & $\mathbf{M}$ & 2 & 60 & - & Abnormal & Normal & $\star$ & + & Propranolol & 480 \\
\hline 38 & 58 & $\mathbf{M}$ & 2 & 72 & - & Abnormal & Enlarged heart & 256 & + & Propranolol & 240 \\
\hline 39 & 62 & $\mathbf{M}$ & 2 & 3 & - & Abnormal & Normal & 200 & + & Practolol & 300 \\
\hline 40 & 49 & $\mathbf{M}$ & 2 & 72 & - & Abnormal & Normal & 256 & + & Propranolol & 320 \\
\hline $4 I$ & 60 & $\mathbf{M}$ & 3 & 2 & + & Abnormal & Normal & 250 & + & Propranolol & 480 \\
\hline 42 & $5 I$ & $\mathbf{M}$ & 2 & 24 & - & Abnormal & Normal & 260 & + & Practolol & 400 \\
\hline 43 & 51 & $\mathbf{M}$ & 2 & 6 & + & Abnormal & Enlarged heart & 310 & + & None & - \\
\hline 44 & 52 & $\mathbf{M}$ & 4 & $\mathbf{I}$ & + & Abnormal & Normal & 250 & + & Propranolol & 120 \\
\hline 45 & 56 & $\mathbf{F}$ & 4 & 12 & + & Abnormal & Normal & 330 & + & Propranolol & 360 \\
\hline 46 & 50 & $\mathbf{M}$ & 3 & 72 & - & Abnormal & Normal & 336 & + & Propranolol & 240 \\
\hline 47 & 46 & $\mathbf{M}$ & 3 & 42 & - & Normal & Normal & 200 & + & Propranolol & 640 \\
\hline 48 & 44 & $\mathbf{M}$ & I & 12 & - & Abnormal & Normal & $\star$ & + & Propranolol & 160 \\
\hline 49 & 42 & $\mathbf{M}$ & 2 & 48 & - & Normal & Normal & 362 & + & Propranolol & 120 \\
\hline
\end{tabular}

* Some cholesterol not measured 
TABLE I (Cont'd)

\begin{tabular}{|c|c|c|c|c|c|c|c|c|c|c|c|}
\hline \multirow{2}{*}{\multicolumn{2}{|c|}{$\begin{array}{l}\text { Case Age } \\
\text { No. (yr) }\end{array}$}} & \multirow[t]{2}{*}{ Sex } & \multicolumn{2}{|l|}{ Angina } & \multirow{2}{*}{$\begin{array}{l}\text { Previous } \\
\text { myo- } \\
\text { cardial } \\
\text { infarct }\end{array}$} & \multirow{2}{*}{$\begin{array}{l}\text { Electro- } \\
\text { cardiogram }\end{array}$} & \multirow{2}{*}{$\begin{array}{l}\text { Chest } \\
\text { radiograph }\end{array}$} & \multirow{2}{*}{$\begin{array}{l}\text { Chole- } \\
\text { sterol } \\
(\mathrm{mg} / \\
100 \mathrm{ml})\end{array}$} & \multirow{2}{*}{$\begin{array}{l}\text { Nitro- } \\
\text { glycerin }\end{array}$} & \multirow{2}{*}{\multicolumn{2}{|c|}{$\begin{array}{l}\text { Beta adrenergic } \\
\text { blocking agents } \\
\qquad \begin{array}{l}\text { Dose/day } \\
(m g)\end{array}\end{array}$}} \\
\hline & & & Grade & $\begin{array}{l}\text { Duration } \\
\text { (mth) }\end{array}$ & & & & & & & \\
\hline 50 & 50 & $\mathbf{M}$ & 3 & 12 & + & Abnormal & Normal & I98 & + & Practolol & 900 \\
\hline 51 & 57 & $\mathbf{M}$ & 3 & 15 & - & Abnormal & Normal & $\star$ & + & Propranolol & 240 \\
\hline 52 & 52 & $\mathbf{M}$ & 2 & 20 & + & Abnormal & Enlarged heart & 282 & + & Practolol & 200 \\
\hline 53 & 50 & $M$ & 2 & 8 & - & Abnormal & Normal & 252 & + & Propranolol & 120 \\
\hline 54 & 37 & $\mathbf{F}$ & 3 & 7 & + & Abnormal & Normal & $\star$ & + & Propranolol & 240 \\
\hline 55 & 42 & $\mathbf{M}$ & 3 & ro & - & Abnormal & Normal & $\star$ & + & Propranolol & 240 \\
\hline 56 & 44 & $\mathbf{M}$ & 3 & 24 & + & Abnormal & Enlarged heart & $\star$ & + & Oxprenolol & 120 \\
\hline 57 & 48 & $\mathbf{M}$ & 2 & 12 & - & Normal & Normal & 286 & + & Practolol & 300 \\
\hline 58 & 47 & $\mathbf{M}$ & 2 & 4 & - & Abnormal & Normal & 266 & + & Propranolol & 120 \\
\hline 59 & 50 & $\mathbf{M}$ & 2 & 72 & - & Normal & Normal & 260 & + & Propranolol & 120 \\
\hline 60 & 40 & $\mathbf{M}$ & I & 12 & - & Normal & Normal & $\star$ & + & Propranolol & 120 \\
\hline $6 I$ & 52 & $M$ & 4 & 12 & - & Abnormal & Normal & I64 & + & Oxprenolol & 120 \\
\hline 62 & 42 & $\mathbf{M}$ & 2 & 8 & + & Abnormal & Enlarged heart & 232 & + & & \\
\hline 63 & 49 & $\mathbf{M}$ & 3 & 13 & - & Abnormal & Normal & 276 & + & Propranolol & 240 \\
\hline 64 & 56 & $\mathbf{M}$ & 3 & 12 & - & Normal & Enlarged heart & 306 & + & & \\
\hline 65 & 45 & $\mathbf{M}$ & 3 & 48 & - & Abnormal & Normal & 260 & + & Practolol & 200 \\
\hline 66 & 40 & $\mathrm{M}$ & 2 & 200 & - & Normal & Normal & 206 & + & Propranolol & 160 \\
\hline 67 & 53 & $\mathbf{M}$ & 3 & 84 & - & Abnormal & Normal & 280 & + & Propranolol & 320 \\
\hline 68 & 54 & $\mathbf{M}$ & 3 & 36 & - & Normal & Normal & $\star$ & + & Practolol & 600 \\
\hline 69 & 51 & $\mathbf{M}$ & 2 & 6 & - & Normal & Normal & $\star$ & + & Practolol & 400 \\
\hline 70 & 60 & $\mathrm{~F}$ & 4 & 8 & + & Abnormal & Normal & 340 & + & Propranolol & 240 \\
\hline 71 & 56 & $\mathbf{M}$ & 2 & 3 & - & Normal & Normal & $\star$ & + & & \\
\hline 72 & 43 & $\mathbf{M}$ & 3 & 8 & - & Normal & Normal & 230 & + & Propranolol & 120 \\
\hline 73 & 61 & $\mathbf{M}$ & 4 & 72 & - & Abnormal & Normal & 272 & + & & \\
\hline 74 & 43 & $\mathbf{M}$ & 3 & 4 & + & Abnormal & Normal & 252 & - & Propranolol & 120 \\
\hline 75 & 49 & F & 3 & 12 & - & Normal & Normal & $\star$ & + & Propranolol & 240 \\
\hline 76 & 48 & $\mathbf{M}$ & 2 & 18 & - & Normal & Normal & I64 & + & & \\
\hline 77 & 39 & $M$ & 4 & 0.2 & - & Abnormal & Normal & & - & & \\
\hline 78 & 31 & $M$ & 4 & 0.5 & - & Abnormal & Normal & & + & & \\
\hline 79 & 38 & $\mathbf{M}$ & I & 24 & - & Normal & Normal & & + & Practolol & 300 \\
\hline 80 & 58 & $\mathbf{M}$ & 4 & 48 & + & Abnormal & Pulm. venous & & & & \\
\hline & & & & & & & congestion & & + & Oxprenolol & 1000 \\
\hline 81 & 47 & $\mathbf{M}$ & 3 & 36 & - & Normal & Normal & & - & & \\
\hline 82 & 64 & $\mathrm{M}$ & 3 & 60 & + & Abnormal & Normal & & + & Propranolol & 240 \\
\hline 83 & 51 & $\mathbf{F}$ & 2 & 6 & - & Abnormal & Normal & & - & Propranolol & 60 \\
\hline 84 & 42 & $\mathbf{M}$ & 4 & $\mathbf{I}$ & - & Abnormal & Enlarged heart & & + & Propranolol & 120 \\
\hline 85 & 38 & $\mathbf{M}$ & 3 & 36 & + & Abnormal & Normal & 310 & + & Oxprenolol & 160 \\
\hline 86 & 55 & $\mathrm{M}$ & 4 & 120 & - & Abnormal & Normal & 258 & + & Practolol & 600 \\
\hline 87 & 62 & $\mathbf{M}$ & 3 & 7 & - & Abnormal & Normal & 246 & + & Propranolol & 240 \\
\hline 88 & 46 & $\mathbf{M}$ & 3 & 108 & - & Normal & Normal & & + & Practolol & 800 \\
\hline 89 & 52 & $\mathbf{M}$ & 3 & 24 & - & Normal & Normal & 390 & + & Propranolol & 80 \\
\hline 90 & 42 & M & 2 & 30 & + & Abnormal & Enlarged heart & 256 & - & Propranolol & 240 \\
\hline 9I & 43 & $\mathbf{M}$ & 2 & 4 & + & Abnormal & Enlarged heart & & + & Practolol & 300 \\
\hline 92 & 34 & $\mathbf{M}$ & 2 & 30 & - & Normal & Normal & 206 & + & & \\
\hline 93 & 45 & $M$ & 3 & 36 & + & Abnormal & Normal & 204 & + & Practolol & 200 \\
\hline 94 & 49 & $\mathbf{M}$ & 3 & 60 & + & Abnormal & Normal & & + & Propranolol & 240 \\
\hline 95 & 48 & $\mathbf{F}$ & 4 & 24 & - & Normal & Normal & & + & Propranolol & 720 \\
\hline 96 & 51 & $\mathbf{M}$ & 4 & 3 & - & Abnormal & Normal & I 88 & + & & \\
\hline 97 & 55 & $M$ & 3 & 48 & + & Abnormal & Normal & & + & Oxprenolol & 120 \\
\hline 98 & 56 & $M$ & 2 & 6 & - & Abnormal & Normal & 270 & + & Propranolol & 240 \\
\hline 99 & 46 & $\mathbf{M}$ & 2 & 3 & + & Abnormal & Normal & & + & Practolol & 600 \\
\hline 100 & $5 I$ & $\mathbf{M}$ & 3 & 4 & - & Abnormal & Normal & 189 & + & Practolol & 300 \\
\hline IOI & 43 & $\mathbf{M}$ & 2 & 2 & - & Abnormal & Normal & 248 & - & Propranolol & 120 \\
\hline 102 & 59 & $\mathbf{M}$ & 2 & 2 & + & Abnormal & Normal & 168 & + & & \\
\hline 103 & 55 & $\mathbf{M}$ & 3 & $\mathbf{I}$ & - & Abnormal & Normal & 234 & - & Practolol & 300 \\
\hline 104 & 56 & $\mathbf{M}$ & 2 & 6 & - & Normal & Normal & 345 & + & Propranolol & 120 \\
\hline 105 & 42 & $\mathbf{M}$ & 2 & 9 & - & Abnormal & Normal & & + & Propranolol & 120 \\
\hline 106 & 49 & $\mathrm{M}$ & 2 & 60 & + & Abnormal & Normal & & + & Propranolol & 120 \\
\hline 107 & 51 & $\mathbf{M}$ & 4 & 4 & - & Abnormal & Normal & & + & Propranolol & 120 \\
\hline 108 & $5 I$ & $\mathbf{M}$ & 3 & 96 & + & Abnormal & Normal & 270 & + & & \\
\hline 109 & $4 \mathrm{I}$ & $\mathbf{M}$ & 4 & 0.5 & - & Normal & Normal & & - & & \\
\hline
\end{tabular}


4). The average grade for the whole series was $2 \cdot 8$. The duration of angina varied from I week to 19 years and averaged 35 months. Eighty-two (76\%) of the patients had an abnormal resting electrocardiogram and there was evidence of previous infarction in $33(30 \%)$. In 16 patients $(15 \%)$ the chest radiograph showed either cardiomegaly or pulmonary venous congestion. The average fasting serum cholesterol ranged from 164 to 540 with an average of $264 \mathrm{mg} / 100 \mathrm{ml}$.

Ninety-eight $(90 \%)$ patients had been treated with sublingual nitroglycerin, and $94(86 \%)$ with beta-adrenergic blocking agents. These drugs were propranolol ( 56 patients, average dose $206 \mathrm{mg}$ per day), practolol (32 patients, average dose $487 \mathrm{mg}$ per day), and oxprenolol ( 6 patients, average dose $280 \mathrm{mg}$ per day).

Table 2 gives the individual results of the preoperative exercise and pacing tests and angiographic assessment.

\section{Exercise test}

Twenty-two patients did not have an exercise test, 9 because they had angina at rest and 13 for other reasons. The maximum work load varied from 150 to 900 , with an average of $488 \mathrm{kpm} / \mathrm{min}$. In 66 the limiting factor was angina or ST depression.

\section{Pacing test}

The resting left ventricular end-diastolic pressure (LVEDP) ranged from 5 to 46 with a mean of 15 mmHg. Seven patients were not paced because of angina at rest at the time of investigation, and in 9 the tests were technically unsatisfactory. Eightyeight $(95 \%)$ of the remaining 93 patients developed angina on pacing, 2 had dyspnoea, and 3 reached the maximum rate of 180 b.p.m. without symptoms.

\section{Angiography}

The individual coronary scores are shown in Table 2. Thirty-nine $(36 \%)$ patients had major lesions (score 3 or 4 ) of a single vessel, $40(37 \%)$ of two, and $30(27 \%)$ of three vessels. Eight $(7 \%)$ patients had a major lesion of the left main coronary artery. The left ventriculogram was normal in $60(55 \%)$ patients, the remainder having varying extent of dyskinesia.

\section{Surgery}

The individual vessels bypassed and the type of graft are indicated in Table 2. Forty-seven patients had a single graft, 45 two grafts, I 6 three grafts, and I four grafts. A prior endarterectomy was done on 7 vessels, and the left internal mammary artery was used 17 times. The details of graft distribution and preoperative flow measurements are shown in Table 3. Flows ranged from 5 to $240 \mathrm{ml} / \mathrm{min}$, with an average of $73 \mathrm{ml} / \mathrm{min}$.

Seven patients died in hospital (Table 2): 3 from pulmonary embolism, 2 from respiratory complications, I from primary ventricular fibrillation, and I from haemorrhage. Of the 3 patients who died of pulmonary embolism, 2 had poor left ventricular function (Cases 28 and 45), and I of these died on the operating table (Case 28); the other 2 occurred late in patients who had been slow to mobilize. All occurred before routine postoperative anticoagulation was used. A third patient with poor left ventricular function (Case 73) died of sudden ventricular fibrillation. Four of the deaths occurred

TABLE 2 Results of investigation

\begin{tabular}{|c|c|c|c|c|c|c|c|c|c|}
\hline \multirow{2}{*}{$\begin{array}{l}\text { Case } \\
\text { No. }\end{array}$} & \multirow{2}{*}{$\begin{array}{l}\text { Maximum } \\
\text { exercise } \\
\text { load } \\
(\mathrm{kpm} / \mathrm{min})\end{array}$} & \multirow{2}{*}{$\begin{array}{l}\text { End } \\
\text { point }\end{array}$} & \multirow{2}{*}{$\begin{array}{l}\text { Left } \\
\text { ventricular } \\
\text { end-diastolic } \\
\text { pressure } \\
(\mathrm{mmHg})\end{array}$} & \multirow{2}{*}{$\begin{array}{l}\text { Maximum } \\
\text { pacing } \\
\text { rate }\end{array}$} & \multirow{2}{*}{$\begin{array}{l}\text { End } \\
\text { point }\end{array}$} & \multicolumn{3}{|c|}{ Coronary arteries: grading of disease } & \multirow{2}{*}{$\begin{array}{l}\text { Left } \\
\text { ventricular } \\
\text { dyskinesia }\end{array}$} \\
\hline & & & & & & $\begin{array}{l}\text { Left } \\
\text { anterior } \\
\text { descending }\end{array}$ & $\begin{array}{l}\text { Left } \\
\text { circumflex }\end{array}$ & Right & \\
\hline I & 450 & Dyspnoea & 28 & I 10 & Angina & $4^{\star}$ & 2 & 4 & 2 \\
\hline 2 & & & 8 & 140 & Angina & 3 & $4^{\star}$ & I & 0 \\
\hline 3 & $\begin{array}{l}\text { Angina at } \\
\text { rest }\end{array}$ & & 46 & 120 & Angina & $4^{\star}$ & $4^{\star}$ & 3 & 2 \\
\hline 4 & 450 & Angina & 10 & 140 & Angina & $3^{\star}$ & $3^{\star}$ & 4 & 0 \\
\hline 5 & 150 & Angina & 12 & 120 & Angina & 2 & $3^{\star}$ & $4^{\star}$ & 0 \\
\hline 6 & 600 & Angina & 16 & 130 & Dyspnoea & I & $3^{\star}$ & 4 & 0 \\
\hline 7 & 450 & Dyspnoea & 5 & 130 & Angina & $2^{\star}$ & $4^{\star}$ & 0 & 0 \\
\hline 8 & 450 & Angina & 10 & 170 & Angina & $2^{\star}$ & $3^{\star}$ & $2^{\star}$ & 0 \\
\hline 9 & & & 14 & 140 & Angina & $2^{\star}$ & $3^{\star}$ & I & 0 \\
\hline ro & 300 & Dyspnoea & 9 & 140 & Angina & $3^{\star}$ & 0 & 0 & 0 \\
\hline II & & & 7 & I 10 & Angina & $3^{\star}$ & 0 & 0 & 0 \\
\hline 12 & 600 & Angina & 22 & 150 & Angina & 3 & $3^{\star}$ & $4^{\star}$ & 0 \\
\hline
\end{tabular}

t, hospital death; $L$, left main stem stenosis $>75$ per cent; $D$, diagonal branch left anterior descending grafted; $\star$, vessel bypassed with saphenous vein; $\oint$, vessel bypassed with left internal mammary artery; $E$, endarterectomy; $\ddagger$, LV surgery. 
TABLE 2 (Cont'd)

\begin{tabular}{|c|c|c|c|c|c|c|c|c|c|}
\hline \multirow{2}{*}{$\begin{array}{l}\text { Case } \\
\text { No. }\end{array}$} & \multirow{2}{*}{$\begin{array}{l}\text { Maximum } \\
\text { exercise } \\
\text { load } \\
(\mathrm{kpm} / \mathrm{min})\end{array}$} & \multirow{2}{*}{$\begin{array}{l}\text { End } \\
\text { point }\end{array}$} & \multirow{2}{*}{$\begin{array}{l}\text { Left } \\
\text { ventricular } \\
\text { en-diastolic } \\
\text { pressure } \\
(\mathrm{mmHg})\end{array}$} & \multirow{2}{*}{$\begin{array}{l}\text { Maximum } \\
\text { pacing } \\
\text { rate }\end{array}$} & \multirow{2}{*}{$\begin{array}{l}\text { End } \\
\text { point }\end{array}$} & \multicolumn{3}{|c|}{ Coronary arteries: grading of disease } & \multirow{2}{*}{$\begin{array}{l}\text { Left } \\
\text { ventricular } \\
\text { dyskinesia }\end{array}$} \\
\hline & & & & & & $\begin{array}{l}\text { Left } \\
\text { anterior } \\
\text { descending }\end{array}$ & $\begin{array}{l}\text { Left } \\
\text { circumflex }\end{array}$ & Right & \\
\hline \multirow[t]{2}{*}{ tr3 } & 600 & $\begin{array}{l}\text { ST de- } \\
\text { pression }\end{array}$ & & & & & & & \\
\hline & & $>2 \mathrm{~mm}$ & 22 & I 10 & Angina & $3^{\star}$ & 3 & $3^{\star}$ & I \\
\hline I4 & $\begin{array}{l}\text { Angina at } \\
\text { rest }\end{array}$ & & 8 & $\begin{array}{l}\text { Angina at } \\
\text { rest }\end{array}$ & & 2 & 2 & $4^{\star}$ & $\mathbf{I}$ \\
\hline \multirow[t]{2}{*}{15} & 600 & $\begin{array}{l}\text { ST de- } \\
\text { pression }\end{array}$ & & & & & & & \\
\hline & & $>2 \mathrm{~mm}$ & 18 & 130 & Angina & $3^{\star}$ & 0 & 3 & 0 \\
\hline 16 & 450 & Angina & Io & I30 & Angina & $3^{\star}$ & 2 & 4 & 0 \\
\hline 17 & 450 & $\begin{array}{l}\text { ST de- } \\
\text { pression } \\
>2 \mathrm{~mm}\end{array}$ & 7 & 140 & Angina & I & I & $4^{\star}$ & I \\
\hline 18 & 150 & Angina & 30 & 120 & Angina & 2 & 2 & $3^{\star}$ & 2 \\
\hline I9 & 450 & $\begin{array}{l}\text { ST de- } \\
\text { pression } \\
>2 \mathrm{~mm}\end{array}$ & I7 & 160 & Angina & $3^{\star}$ & $3^{\star}$ & $4^{\star}$ & \\
\hline 20 & 300 & Angina & 25 & 140 & Angina & $3^{\star}$ & I & $\begin{array}{l}4 \\
0\end{array}$ & $\begin{array}{l}3 \\
0\end{array}$ \\
\hline $2 I$ & 600 & Angina & 20 & 140 & Angina & 4 & $3^{\star}$ & $4^{\star}$ & 3 \\
\hline 22 & 750 & Angina & 20 & I50 & Angina & 3 & I & $4^{\star}$ & 0 \\
\hline 23 & 300 & Angina & 8 & 170 & Angina & $3^{\star}$ & 2 & I & 0 \\
\hline 24 & 150 & Angina & I5 & 150 & Angina & $4^{\star}$ & I & 2 & $\mathbf{I}$ \\
\hline 25 & & & 5 & & & $4^{\star}$ & 0 & 0 & I \\
\hline 26 & 300 & Angina & I5 & 120 & Angina & 0 & $3^{\star}$ & 0 & 0 \\
\hline \multirow{2}{*}{$\begin{array}{r}27 \\
+28\end{array}$} & 450 & Angina & 8 & 140 & Angina & 2 & $3^{\star}$ & I & 2 \\
\hline & $\begin{array}{l}\text { Angina at } \\
\text { rest }\end{array}$ & & 17 & $\begin{array}{l}\text { Angina at } \\
\text { rest }\end{array}$ & & $3^{\star}$ & 3 & $4^{\star}$ & 3 \\
\hline 29 & $\begin{array}{c}\text { Angina at } \\
\text { rest }\end{array}$ & & 7 & $\begin{array}{c}\text { Angina at } \\
\text { rest }\end{array}$ & & 0 & $3^{\star}$ & $\mathbf{I}$ & \\
\hline \multirow[t]{2}{*}{30} & 450 & $\begin{array}{l}\text { ST de- } \\
\text { pression }\end{array}$ & & & & & & & \\
\hline & Angina at & $>2 \mathrm{~mm}$ & $\begin{array}{l}10 \\
16\end{array}$ & $\begin{array}{l}\text { I60 } \\
\text { Angina at }\end{array}$ & Angina & $3^{\star}$ & 0 & I & 0 \\
\hline $3 I$ & rest & & & rest & & $4^{\star}$ & 2 & I & 0 \\
\hline \multirow[t]{2}{*}{32} & 450 & $\begin{array}{l}\text { Angina, } \\
\text { ST de- } \\
\text { pression }\end{array}$ & & & & & & & \\
\hline & & $>2 \mathrm{~mm}$ & 17 & I5O & Angina & $4^{\star}$ & 3 & 3 & I \\
\hline 33 & 300 & Angina & 12 & 140 & Angina & $4^{\star}$ & 4 & $2^{\star}$ & I \\
\hline 34 & 300 & Angina & II & 170 & Angina & 3 & 0 & $4^{\star}$ & I \\
\hline 35 & 300 & Dyspnoea & 14 & 130 & Angina & $3^{\star}$ & I & $4^{\star}$ & 2 \\
\hline 36 & 450 & Angina & 15 & 150 & Angina & $3^{\star}$ & 4 & $4^{\star}$ & 4 \\
\hline 37 & & & 33 & & & $3^{\star}$ & I & 0 & 0 \\
\hline+38 & 450 & Angina & 19 & 120 & Angina & $2^{\star}$ & 0 & $3^{\star}$ & 0 \\
\hline 39 & & & 8 & 140 & Angina & $3^{\star}$ & $3^{\star}$ & 0 & 0 \\
\hline 40 & & & 12 & & & $3^{\star}$ & 3 & $4^{\star}$ & 2 \\
\hline \multirow[t]{2}{*}{$4 I$} & 450 & $\begin{array}{l}\text { Angina, } \\
\text { ST de- } \\
\text { pression }\end{array}$ & & & & & & & \\
\hline & & $>2 \mathrm{~mm}$ & 9 & I50 & Angina & $3^{\star}$ & I & $3^{\star}$ & $\mathbf{I}$ \\
\hline+42 & 450 & Dyspnoea & 12 & 170 & Angina & $3 *$ & $3^{\star}$ & I & 0 \\
\hline 43 & & & I5 & 120 & Angina & $3^{\star}$ & 4 & 3 & 3 \\
\hline \multirow{4}{*}{$\begin{array}{r}44 \\
+45 \\
46\end{array}$} & 450 & Fatigue & 8 & 160 & Angina & $4^{\star}$ & 2 & $3^{\star}$ & 3 \\
\hline & & & 15 & 140 & Angina & 3 & I & $3^{\star}$ & 3 \\
\hline & 450 & $\begin{array}{l}\text { ST de- } \\
\text { pression }\end{array}$ & & & & & & & \\
\hline & & $>2 \mathrm{~mm}$ & 7 & I 20 & Angina & $3^{\star}$ & I & 3 & 0 \\
\hline \multirow{3}{*}{$\begin{array}{l}47 \\
48\end{array}$} & 300 & Dyspnoea & 9 & & & $D * 2$ & $2^{\star}$ & 3 & 0 \\
\hline & 750 & $\begin{array}{l}\text { ST de- } \\
\text { pression }\end{array}$ & & & & & & & \\
\hline & & $>2 \mathrm{~mm}$ & 13 & & & $3^{\star}$ & 0 & 2 & 0 \\
\hline
\end{tabular}


846 Balcon, Honey, Rickards, Sturridge, Walsh, Wilkinson, and Wright

TABLE 2 (Cont'd)

\begin{tabular}{|c|c|c|c|c|c|c|c|c|c|c|}
\hline \multirow{2}{*}{$\begin{array}{l}\text { Case } \\
\text { No. }\end{array}$} & \multirow{2}{*}{$\begin{array}{l}\text { Maximum } \\
\text { exercise } \\
\text { load } \\
\text { (kpm/min) }\end{array}$} & \multirow{2}{*}{$\begin{array}{l}\text { End } \\
\text { point }\end{array}$} & \multirow{2}{*}{$\begin{array}{l}\text { Left } \\
\text { ventricular } \\
\text { end-diastolic } \\
\text { pressure } \\
(\mathrm{mmHg})\end{array}$} & \multirow{2}{*}{$\begin{array}{l}\text { Maximum } \\
\text { pacing } \\
\text { rate }\end{array}$} & \multirow{2}{*}{$\begin{array}{l}\text { End } \\
\text { point }\end{array}$} & \multicolumn{4}{|c|}{ Coronary arteries: grading of disease } & \multirow{2}{*}{$\begin{array}{l}\text { Left } \\
\text { ventricular } \\
\text { dyskinesia }\end{array}$} \\
\hline & & & & & & $\begin{array}{l}\text { Left } \\
\text { ante } \\
\text { desct }\end{array}$ & $\begin{array}{l}\text { erior } \\
\text { ending }\end{array}$ & $\begin{array}{l}\text { Left } \\
\text { circumflex }\end{array}$ & Right & \\
\hline 49 & 600 & Angina & 5 & 150 & Angina & & $4^{\star}$ & $4^{\star}$ & 2 & o \\
\hline 50 & 300 & Angina & 10 & 170 & Angina & & $3^{\star}$ & 0 & $\mathbf{I}$ & o \\
\hline 51 & 450 & Angina & 18 & IIO & Angina & & $3^{\star}$ & 4 & $3^{\star}$ & 2 \\
\hline 52 & 750 & Dyspnoea & 14 & 150 & Angina & $D^{\star}$ & $4^{\star}$ & 0 & $4^{\star}$ & 0 \\
\hline \multirow[t]{2}{*}{53} & 750 & $\begin{array}{l}\text { Angina, } \\
\text { ST de- } \\
\text { pression }\end{array}$ & & & & & & & & \\
\hline & & $>2 \mathbf{m m}$ & I6 & 150 & Angina & & $4^{\star}$ & 2 & 2 & o \\
\hline \multirow{3}{*}{$\begin{array}{l}54 \\
55\end{array}$} & 300 & Dyspnoea & Io & 150 & Angina & & $4^{\star}$ & 3 & 2 & I \\
\hline & 600 & $\begin{array}{l}\mathrm{ST} \text { de- } \\
\text { pression }\end{array}$ & & & & & & & & \\
\hline & & $>2 \mathrm{~mm}$ & 10 & 130 & Angina & DS & 2 & $3^{\star}$ & 0 & 0 \\
\hline \multirow[t]{2}{*}{56} & 300 & $\begin{array}{l}\text { ST de- } \\
\text { pression }\end{array}$ & & & & & & & & \\
\hline & & $>2 \mathrm{~mm}$ & 6 & 160 & Angina & & $4^{\star}$ & 2 & 2 & 2 \\
\hline \multirow{6}{*}{$\begin{array}{l}57 \\
58 \\
59 \\
60 \\
61\end{array}$} & 600 & Angina & 7 & I IO & Angina & L & $3^{\star}$ & $3^{\star}$ & 3 & 2 \\
\hline & 450 & Angina & 7 & 180 & Angina & DS & $3^{\star}$ & $2^{\star}$ & 0 & 0 \\
\hline & 450 & Fatigue & 15 & Iro & Angina & & I & 3 & $3^{\star}$ & 0 \\
\hline & 900 & Fatigue & 13 & 140 & Angina & & $4^{\star}$ & $3^{\star}$ & $3^{\star}$ & I \\
\hline & Angina at & & & & & & & & & \\
\hline & rest & & IO & 130 & Angina & L & $3^{\star}$ & $\circ$ & $3^{\star}$ & 2 \\
\hline 62 & 600 & Angina & 23 & 140 & Angina & & 4 & $2^{\star}$ & 3 & $3 \ddagger$ \\
\hline 63 & 450 & $\begin{array}{l}\text { Angina, } \\
\text { ST de- } \\
\text { pression } \\
>2 \mathrm{~mm}\end{array}$ & 14 & $\begin{array}{l}\text { Angina at } \\
\text { rest }\end{array}$ & & & $4^{\star}$ & $3^{\star}$ & $4^{\star}$ & 0 \\
\hline \multirow[t]{3}{*}{64} & 450 & Angina, & & & & & & & & \\
\hline & & pression & & & & & & & & \\
\hline & & $>2 \mathrm{~mm}$ & 15 & 180 & Angina & & $4^{\star}$ & $4^{\star}$ & $3^{\star}$ & I \\
\hline \multirow[t]{2}{*}{65} & 600 & $\begin{array}{l}\text { Angina, } \\
\text { ST de- }\end{array}$ & & & & & & & & \\
\hline & & $>2 \mathrm{~mm}$ & 15 & 160 & Dyspnoea & & $4^{\star}$ & $3^{\star}$ & 3 & I \\
\hline 66 & 600 & Angina & 12 & 150 & Angina & $D^{\star}$ & 3 & $3^{\star}$ & 4 & I \\
\hline 67 & 300 & Angina & 15 & 100 & Angina & & $3 \delta$ & 3 & $4^{\star}$ & 2 \\
\hline 68 & 600 & Dyspnoea & 17 & 140 & Angina & & $3 \rrbracket$ & 2 & 0 & 0 \\
\hline \multirow[t]{3}{*}{69} & 450 & Angina, & & & & & & & & \\
\hline & & pression & & & & & & & & \\
\hline & & $>2 \mathrm{~mm}$ & 15 & 170 & Angina & & 4 & 0 & $3^{\star}$ & 0 \\
\hline 70 & 450 & Dyspnoea & 13 & 130 & Angina & & $4^{\star}$ & 0 & 0 & 0 \\
\hline 71 & 450 & Angina & I2 & IIO & Angina & & 3. & 0 & 0 & 0 \\
\hline 72 & 600 & Dyspnoea & 16 & I 70 & Angina & $D^{\star}$ & $3^{\star}$ & 0 & 0 & 0 \\
\hline \multirow[t]{2}{*}{ t73 } & 450 & Angina & 15 & Angina at & & & & & & \\
\hline & & & & rest & & $D^{\star}$ & $2^{\star}$ & 3 & $3^{\star}$ & 2 \\
\hline \multirow[t]{2}{*}{74} & 450 & $\begin{array}{l}\text { Angina, } \\
\text { ST de- }\end{array}$ & & & & & & & & \\
\hline & & $\begin{array}{l}\text { pression } \\
>2 \mathrm{~mm}\end{array}$ & Io & 130 & Angina & & $4^{\star}$ & 0 & 2 & I \\
\hline & 300 & Angina & 18 & 140 & Angina & & $\begin{array}{l}4 \\
0\end{array}$ & 0 & $3^{\star}$ & 0 \\
\hline \multirow[t]{2}{*}{76} & 750 & $\begin{array}{l}\text { ST de- } \\
\text { pression }\end{array}$ & & & & & & & & \\
\hline & & $>2 \mathrm{~mm}$ & II & 180 & Angina & & $3^{\star}$ & 2 & 2 & o \\
\hline 77 & $\begin{array}{l}\text { Angina at } \\
\text { rest }\end{array}$ & & II & $\begin{array}{c}\text { Angina at } \\
\text { rest }\end{array}$ & & $D^{\star}$ & $3^{\star}$ & $3 \star$ & $3^{\star}$ & 0 \\
\hline \multirow[t]{2}{*}{78} & Angina at & & & & & & & & & \\
\hline & rest & & 13 & 180 & & $D^{\star}$ & $3^{\star}$ & 0 & 2 & 0 \\
\hline
\end{tabular}


TABLE 2 (Cont'd)

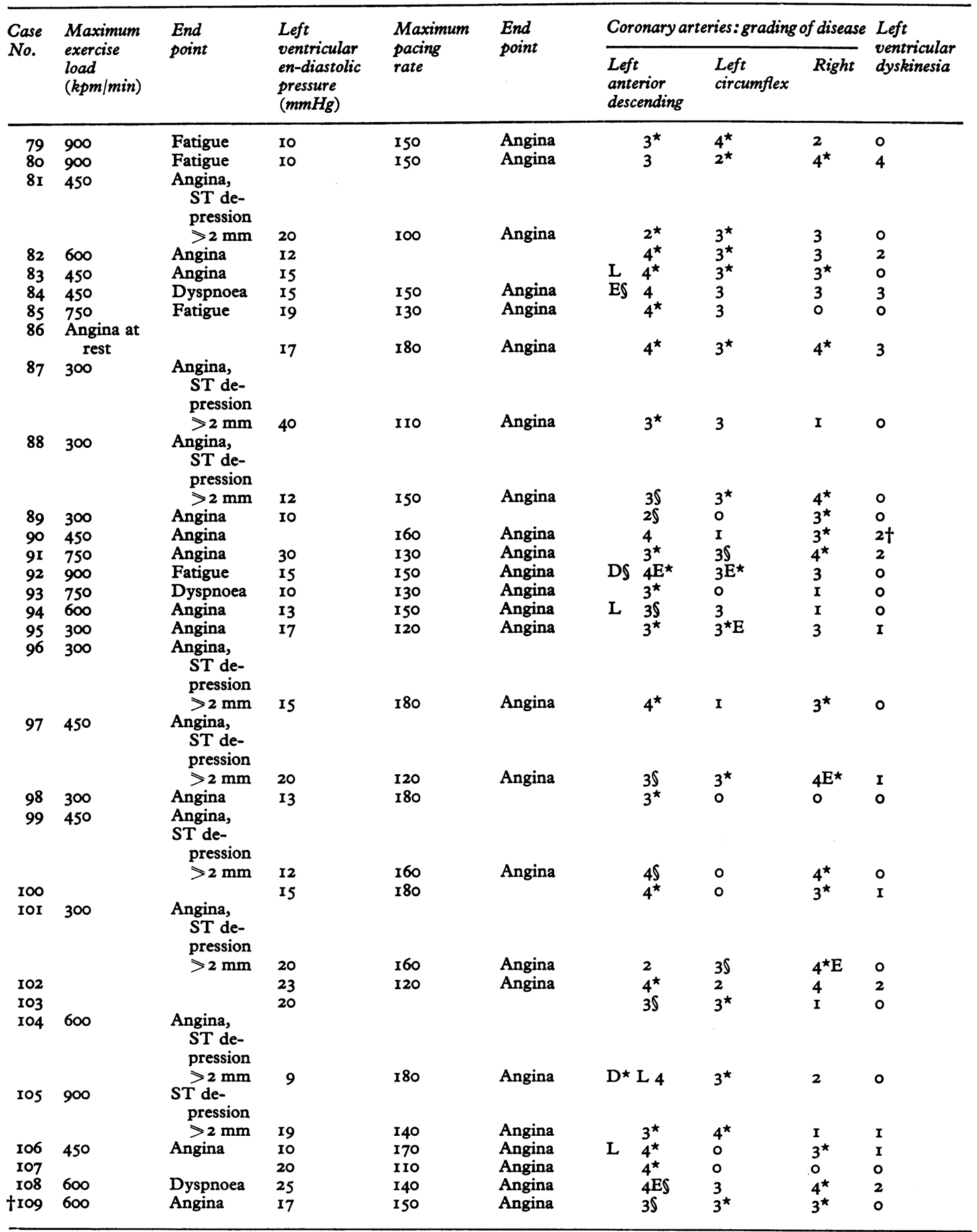


TABLE 3 Graft distribution and flow

\begin{tabular}{|c|c|c|c|c|c|}
\hline \multirow[t]{2}{*}{ Site of anastomosis } & \multirow[t]{2}{*}{ Graft used } & \multirow[t]{2}{*}{$\begin{array}{l}\text { No. of } \\
\text { patients }\end{array}$} & \multicolumn{3}{|l|}{$\begin{array}{l}\text { Flow } \\
(\mathrm{ml} / \mathrm{min})\end{array}$} \\
\hline & & & Average & Range & $\begin{array}{l}\text { No. of } \\
\text { patients }\end{array}$ \\
\hline Left anterior descending & $\left\{\begin{array}{l}\text { Vein } \\
\text { Artery }\end{array}\right.$ & $\begin{array}{l}72 \\
13\end{array}$ & $\begin{array}{l}78 \\
63\end{array}$ & $\begin{array}{r}5-240 \\
20-140\end{array}$ & $\begin{array}{r}52 \\
7\end{array}$ \\
\hline Diagonal & $\begin{array}{l}\text { Vein } \\
\text { Artery }\end{array}$ & $\begin{array}{l}9 \\
2\end{array}$ & $\begin{array}{l}46 \\
50\end{array}$ & $17-80$ & $\begin{array}{l}8 \\
\mathrm{I}\end{array}$ \\
\hline Circumflex & $\begin{array}{l}\text { Vein } \\
\text { Artery }\end{array}$ & $\begin{array}{r}44 \\
2\end{array}$ & 72 & $10-160$ & 21 \\
\hline Right coronary artery & Vein & 47 & 77 & $7-150$ & 27 \\
\hline Totals & & 189 & 73 & $5-240$ & II6 \\
\hline
\end{tabular}

in patients with grade 0 or I preoperative left ventricular angiograms, Cases 13 and 38 of respiratory complications, Case 42 from pulmonary embolism, and Case 109 from haemorrhage. Of the IO2 survivors, 7 developed fresh $Q$ waves on the postoperative electrocardiogram.

\section{Postoperative follow-up}

There have been no deaths in the 102 patients who left hospital and have been followed for up to 34 months. Five patients have had a documented myocardial infarction, one of whom (Case 3I) had a further bypass operation. Postoperative $T$ wave inversion has persisted in a further 23 patients.

The exercise performance frequently improved considerably between the first and second postoperative visits and this was attributed to recovery

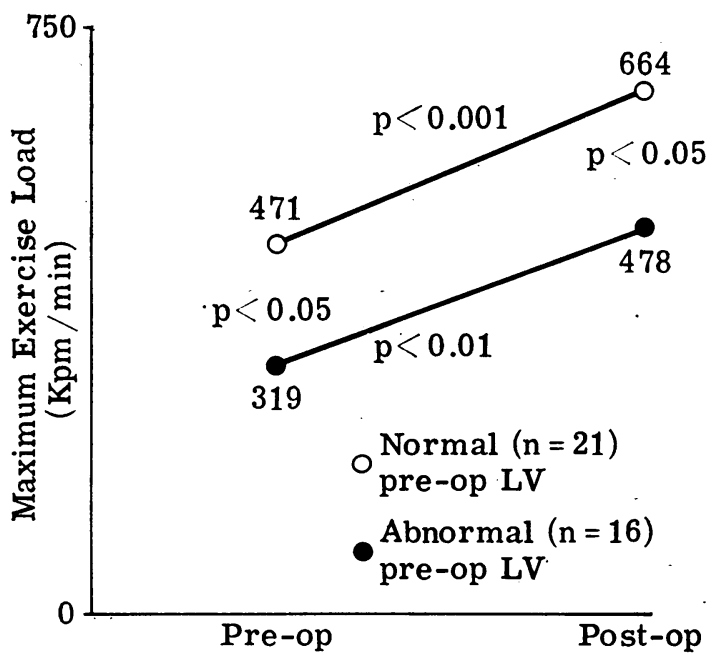

FIG. I The results of pre- and postoperative exercise tests. from the residual effects of major surgery. The follow-up data of the 50 patients who have been followed for more than 6 months (average 13.9 months) are therefore presented. Thirty-six (72\%) of them have had no further angina; this has improved by at least one clinical grade in a further I2 $(24 \%)$ patients and $2(4 \%)$ patients are unchanged. The average grade of angina has fallen from $2 \cdot 8$ to 0.5 . Fig. I shows the results of the preoperative and the latest postoperative exercise test in the 37 patients in whom data are available. Twenty-one patients with a normal preoperative ventriculogram increased their performance from an average of $47 \mathrm{I}$ $\mathrm{kpm} / \mathrm{min}$ to $664 \mathrm{kpm} / \mathrm{min}(\mathrm{P}<0.00 \mathrm{I})$. The 16 patients with abnormal ventriculograms started out at a significantly $(P<0.05)$ lower average level, $319 \mathrm{kpm} / \mathrm{min}$, but improved their exercise performance by a similar amount to an average of $478 \mathrm{kpm} / \mathrm{min}(\mathrm{P}<0.0 \mathrm{I})$.

\section{Postoperative reinvestigations}

Table 4 compares the results of the pre- and postoperative studies in the 33 patients who were reinvestigated, at an average of 8 months after operation. The group includes 12 patients who either had angina or who had an attack of chest pain at rest without having evidence of infarction, and one who had an infarct (Case 3I) and later had a second operation to bypass a new lesion in the circumflex artery and gave their informed consent to reinvestigation. The remaining 20 were free of angina. Fig. 2 compares the pre- and postoperative distribution of the clinical grading and the results of exercise and pacing tests. Of the 7 patients whose clinical angina persisted after operation, 4 had an occluded graft. The exercise performance increased from an average of $394 \mathrm{kpm} / \mathrm{min}$ to an average of $587 \mathrm{kpm} / \mathrm{min}(\mathrm{P}<0.0 \mathrm{I}) ; 28$ of the $3 \mathrm{I}$ patients were limited by angina or ST depression before opera- 
TABLE 4 Comparison of pre- and postoperative investigation

\begin{tabular}{|c|c|c|c|c|c|c|c|c|c|c|c|c|c|}
\hline \multirow[t]{2}{*}{$\begin{array}{l}\text { Case } \\
\text { No. }\end{array}$} & \multicolumn{2}{|c|}{ Angina grade } & \multicolumn{2}{|c|}{$\begin{array}{l}\text { Maximum } \\
\text { exercise load } \\
\text { and end point }\end{array}$} & \multicolumn{2}{|c|}{$\begin{array}{l}\text { Maximum pacing } \\
\text { rate and end } \\
\text { point }\end{array}$} & \multicolumn{2}{|c|}{$\begin{array}{l}\text { Left ventricular } \\
\text { end-diastolic } \\
\text { pressure }\end{array}$} & \multicolumn{2}{|c|}{$\begin{array}{l}\text { Left ventricular } \\
\text { dyskinesia score }\end{array}$} & \multicolumn{2}{|c|}{$\begin{array}{l}\text { Total } \\
\text { coronary } \\
\text { score }\end{array}$} & \multirow[t]{2}{*}{$\begin{array}{l}\text { Graft } \\
\text { patency }\end{array}$} \\
\hline & Pre & Post & Pre & Post & Pre & Post & Pre & Post & Pre & Post & Pre & Post & \\
\hline $\mathbf{I}$ & 4 & 3 & $\begin{array}{l}450, \\
\text { dys- } \\
\text { pnoea }\end{array}$ & $\begin{array}{l}300 \text {, } \\
\text { angina }\end{array}$ & $\begin{array}{l}\text { I Io, } \\
\text { angina }\end{array}$ & $160 A$ & 28 & 17 & 2 & & Io & 9 & $\bullet$ \\
\hline 2 & 3 & 0 & & & $\begin{array}{l}\text { I40, } \\
\text { angina }\end{array}$ & 180 & 8 & I5 & 0 & 0 & 8 & & 0 \\
\hline 4 & 3 & 0 & $\begin{array}{l}\text { 450, } \\
\text { angina }\end{array}$ & $\begin{array}{l}750, \mathrm{ST} \\
\text { de- } \\
\text { pression } \\
>2 \mathrm{~mm}\end{array}$ & $\begin{array}{l}\text { I40, } \\
\text { angina }\end{array}$ & I70 & 8 & 10 & 0 & & ro & & $\begin{array}{l}0 \\
0 \\
0\end{array}$ \\
\hline 5 & 3 & 0 & $\begin{array}{l}\text { I50, } \\
\text { angina }\end{array}$ & $\begin{array}{l}600 \\
\text { fatigua }\end{array}$ & $\begin{array}{l}\text { 120, } \\
\text { angina }\end{array}$ & 180 & 12 & 16 & 0 & 0 & 9 & 8 & $\begin{array}{l}0 \\
0\end{array}$ \\
\hline 12 & 2 & 0 & $\begin{array}{l}600, \\
\text { angina }\end{array}$ & $\begin{array}{l}300, \\
\text { angina }\end{array}$ & $\begin{array}{l}\text { I50, } \\
\text { angina }\end{array}$ & 180 & 22 & 33 & 0 & & Io & 8 & $\begin{array}{l}0 \\
0\end{array}$ \\
\hline 14 & 4 & 3 & $\begin{array}{l}\text { Angina } \\
\text { at rest }\end{array}$ & $\begin{array}{l}300, \\
\text { angina }\end{array}$ & $\begin{array}{l}\text { Angina } \\
\text { at rest }\end{array}$ & . & 8 & 7 & $\mathbf{I}$ & 0 & 8 & 8 & $\bullet$ \\
\hline 15 & 2 & 0 & $\begin{array}{l}600, \\
\text { angina }\end{array}$ & $\begin{array}{l}600, \\
\text { fatigue }\end{array}$ & $\begin{array}{l}\text { x30, } \\
\text { angina }\end{array}$ & 180 & I8 & 5 & 0 & 0 & 6 & 5 & 0 \\
\hline 16 & 3 & 0 & $\begin{array}{l}450, \\
\text { angina }\end{array}$ & $\begin{array}{l}600, \\
\text { fatigue }\end{array}$ & $\begin{array}{l}\text { I30, } \\
\text { angina }\end{array}$ & 180 & 13 & 15 & 0 & 0 & Io & II & $\stackrel{\bullet}{0}$ \\
\hline 17 & 3 & 0 & $\begin{array}{l}\text { 450, } \mathrm{ST} \\
\text { de- } \\
\text { pression } \\
>2 \mathrm{~mm}\end{array}$ & $\begin{array}{l}450, \\
\text { fatigue } \\
n\end{array}$ & $\begin{array}{l}\text { I40, } \\
\text { angina }\end{array}$ & 180 & 7 & 7 & I & & 6 & 5 & O \\
\hline I8 & 3 & 2 & $\begin{array}{l}\text { I50, } \\
\text { angina }\end{array}$ & $\begin{array}{l}\text { 600, } \\
\text { fatigue }\end{array}$ & $\begin{array}{l}\text { 120, } \\
\text { angina }\end{array}$ & I70 & 30 & 25 & 2 & & 7 & 8 & O \\
\hline 19 & 3 & 0 & $\begin{array}{l}\text { 450, } \mathrm{ST} \\
\text { de- } \\
\text { pression } \\
>2 \mathrm{~mm}\end{array}$ & $\begin{array}{l}750, \\
\text { fatigue } \\
\text { n }\end{array}$ & $\begin{array}{l}\text { 160, } \\
\text { angina }\end{array}$ & 180 & 17 & 12 & 3 & & IO & & $\begin{array}{l}0 \\
0 \\
0 \\
0\end{array}$ \\
\hline 20 & 2 & 0 & $\begin{array}{l}300, \\
\text { angina }\end{array}$ & $\begin{array}{l}450 \\
\text { dys- } \\
\text { pnoea }\end{array}$ & $\begin{array}{l}\text { I40, } \\
\text { angina }\end{array}$ & 180 & 25 & 13 & 0 & 0 & 4 & 4 & 0 \\
\hline $2 I$ & 2 & 0 & $\begin{array}{l}\text { 600, } \\
\text { angina }\end{array}$ & $\begin{array}{l}600, \\
\text { dys- } \\
\text { pnoea }\end{array}$ & $\begin{array}{l}\text { I40, } \\
\text { angina }\end{array}$ & $\begin{array}{l}\text { 160, } \\
\text { angina }\end{array}$ & 20 & 28 & 3 & 4 & I I & I I & $\stackrel{0}{0}$ \\
\hline 22 & 3 & 0 & $\begin{array}{l}750, \\
\text { angina }\end{array}$ & $\begin{array}{l}600, \\
\text { fatigue }\end{array}$ & $\begin{array}{l}\text { I50, } \\
\text { angina }\end{array}$ & & 20 & 7 & 0 & 0 & 8 & 6 & 0 \\
\hline 23 & 3 & 0 & $\begin{array}{l}300, \\
\text { angina }\end{array}$ & $\begin{array}{l}750, \\
\text { fatigue }\end{array}$ & $\begin{array}{l}\text { I70, } \\
\text { angina }\end{array}$ & 180 & 8 & II & 0 & 0 & 6 & 6 & 0 \\
\hline 24 & 3 & 3 & $\begin{array}{l}\text { I50, } \\
\text { angina }\end{array}$ & $\begin{array}{l}300, \\
\text { angina }\end{array}$ & $\begin{array}{l}\text { I50, } \\
\text { angina }\end{array}$ & $\begin{array}{l}\text { I40, } \\
\text { angina }\end{array}$ & 15 & I I & I & I & 7 & 7 & 0 \\
\hline 25 & 3 & 0 & & $\begin{array}{l}750, \\
\text { fatigue }\end{array}$ & & & 5 & I4 & I & 0 & 4 & 7 & 0 \\
\hline 29 & 3 & 0 & $\begin{array}{l}\text { Angina } \\
\text { at rest }\end{array}$ & $\begin{array}{l}600, \\
\text { dys- } \\
\text { pnoea }\end{array}$ & $\begin{array}{l}\text { Angina } \\
\text { at rest }\end{array}$ & $\begin{array}{l}\text { I50, } \\
\text { angina }\end{array}$ & 7 & 12 & & & 4 & 6 & 0 \\
\hline 30 & 2 & 0 & $\begin{array}{l}\text { 450, } \\
\text { ST } \\
\text { de- } \\
\text { pression } \\
>2 \mathrm{~mm}\end{array}$ & $\begin{array}{l}\text { I050, } \\
\text { fatigue } \\
\text { nn }\end{array}$ & $\begin{array}{l}160 \\
\text { angina }\end{array}$ & 180 & 10 & 12 & 0 & 0 & 4 & 7 & 0 \\
\hline 31 & 4 & 2 & $\begin{array}{l}\text { Angina } \\
\text { at rest }\end{array}$ & $\begin{array}{l}600, \\
\text { dys- } \\
\text { pnoea }\end{array}$ & $\begin{array}{l}\text { Angina } \\
\text { at rest }\end{array}$ & $\begin{array}{l}\text { I60, } \\
\text { angina }\end{array}$ & I6 & 22 & 0 & 2 & 7 & IO & 0 \\
\hline 32 & 3 & I & $\begin{array}{l}\text { 450, } \\
\text { ST } \\
\text { pression } \\
>2 \mathrm{~mm}\end{array}$ & $\begin{array}{l}750, \\
\quad \text { angina } \\
n\end{array}$ & $\begin{array}{l}\text { I50, } \\
\text { angina }\end{array}$ & $\begin{array}{l}\text { I60, } \\
\text { angina }\end{array}$ & 17 & 15 & I & I & IO & II & 0 \\
\hline
\end{tabular}


TABLE 4 (Cont'd)

\begin{tabular}{|c|c|c|c|c|c|c|c|c|c|c|c|c|c|}
\hline \multirow[t]{2}{*}{$\begin{array}{l}\text { Case } \\
\text { No. }\end{array}$} & \multicolumn{2}{|c|}{ Angina grade } & \multicolumn{2}{|c|}{$\begin{array}{l}\text { Maximum } \\
\text { exercise load } \\
\text { and end point }\end{array}$} & \multicolumn{2}{|c|}{$\begin{array}{l}\text { Maximum pacing } \\
\text { rate and end } \\
\text { point }\end{array}$} & \multicolumn{2}{|c|}{$\begin{array}{l}\text { Left ventricular } \\
\text { end-diastolic } \\
\text { pressure }\end{array}$} & \multicolumn{2}{|c|}{$\begin{array}{l}\text { Left ventricular } \\
\text { dyskinesia score }\end{array}$} & \multicolumn{2}{|c|}{$\begin{array}{l}\text { Total } \\
\text { coronary } \\
\text { score }\end{array}$} & \multirow[t]{2}{*}{$\begin{array}{l}\text { Graft } \\
\text { patency }\end{array}$} \\
\hline & Pre & Post & Pre & Post & Pre & Post & Pre & Post & Pre & Post & Pre & Post & \\
\hline 33 & 3 & 0 & $\begin{array}{l}300, \\
\text { angina }\end{array}$ & $\begin{array}{l}750, \\
\text { fatigue }\end{array}$ & $\begin{array}{l}\text { I40, } \\
\text { angina }\end{array}$ & $\begin{array}{l}\text { I70, } \\
\text { angina }\end{array}$ & 12 & I5 & $\mathbf{I}$ & I & IO & IO & $\stackrel{0}{\bullet}$ \\
\hline $34^{\circ}$ & 3 & 0 & $\begin{array}{l}300, \\
\text { angina }\end{array}$ & $\begin{array}{l}600, \\
\text { ST } \\
\text { de- } \\
\text { pression } \\
>2 \mathrm{~mm}\end{array}$ & $\begin{array}{l}\text { I70, } \\
\text { angina }\end{array}$ & 180 & II & 12 & I & I & 8 & IO & 0 \\
\hline 36 & 3 & 0 & $\begin{array}{l}450 \text {, } \\
\text { angina }\end{array}$ & $\begin{array}{l}\text { 300, } \\
\text { dys- } \\
\text { pnoea }\end{array}$ & $\begin{array}{l}\text { I50, } \\
\text { angina }\end{array}$ & & 7 & IO & 4 & 4 & I I & 9 & $\begin{array}{l}0 \\
0\end{array}$ \\
\hline $4 I$ & 3 & 3 & $\begin{array}{l}450, \\
\text { angina }\end{array}$ & $\begin{array}{l}450 \text {, } \\
\text { angina }\end{array}$ & $\begin{array}{l}\text { I5O, } \\
\text { angina }\end{array}$ & $\begin{array}{l}\text { I20, } \\
\text { angina }\end{array}$ & 9 & II & I & 4 & 7 & II & $\begin{array}{l}0 \\
0\end{array}$ \\
\hline 49 & 2 & 0 & $\begin{array}{l}600, \\
\text { angina }\end{array}$ & $\begin{array}{l}900, \\
\text { fatigue }\end{array}$ & $\begin{array}{l}\text { 150, } \\
\text { angina }\end{array}$ & 180 & 5 & 9 & 0 & 0 & 8 & 9 & $\begin{array}{l}0 \\
0\end{array}$ \\
\hline 52 & 2 & 0 & $\begin{array}{l}750, \\
\text { dys- } \\
\text { pnoea }\end{array}$ & $\begin{array}{l}\text { 450, } \\
\text { dys- } \\
\text { pnoea }\end{array}$ & $\begin{array}{l}\text { I50, } \\
\text { angina }\end{array}$ & & I4 & I0 & 0 & I & 8 & II & $\begin{array}{l}0 \\
0 \\
0\end{array}$ \\
\hline 53 & 2 & 0 & $\begin{array}{l}\text { 750, } \\
\text { ST } \\
\text { de- } \\
\text { pression }\end{array}$ & $\begin{array}{l}900, \\
\text { fatigue }\end{array}$ & $\begin{array}{l}\text { I50, } \\
\text { angina }\end{array}$ & 180 & I6 & 12 & 0 & 0 & 8 & 8 & 0 \\
\hline 59 & 2 & 0 & $\begin{array}{l}450, \\
\text { fatigue }\end{array}$ & $\begin{array}{l}450 \text {, } \\
\text { angina }\end{array}$ & $\begin{array}{l}\text { I IO, } \\
\text { angina }\end{array}$ & & I5 & I2 & 0 & 0 & 7 & & 0 \\
\hline 62 & 2 & 0 & $\begin{array}{l}600, \\
\text { angina }\end{array}$ & $\begin{array}{l}\text { 450, } \\
\text { dys- } \\
\text { pnoea }\end{array}$ & $\begin{array}{l}\text { I40, } \\
\text { angina }\end{array}$ & 180 & 23 & 30 & 3 & 4 & 9 & IO & - \\
\hline 77 & 4 & 0 & $\begin{array}{l}\text { Angina } \\
\text { at rest }\end{array}$ & $\begin{array}{l}750, \\
\text { ST } \\
\text { de- } \\
\text { pression } \\
>2 \mathrm{~mm}\end{array}$ & $\begin{array}{l}\text { Angina } \\
\text { at rest }\end{array}$ & $\begin{array}{l}\text { I60, } \\
\text { angina }\end{array}$ & II & 12 & $\mathbf{I}$ & I & 9 & IO & $\begin{array}{l}0 \\
0\end{array}$ \\
\hline 78 & 4 & 0 & $\begin{array}{l}\text { Angina } \\
\text { at rest }\end{array}$ & $\begin{array}{l}750, \\
\text { dys- } \\
\text { pnoea }\end{array}$ & $\begin{array}{l}\text { I80, } \\
\text { angina }\end{array}$ & $\begin{array}{l}\text { I70, } \\
\text { angina }\end{array}$ & I3 & 14 & 0 & 0 & 5 & 5 & ○ \\
\hline 84 & 4 & 0 & $\begin{array}{l}\text { Angina } \\
\text { at rest }\end{array}$ & $\begin{array}{l}450, \\
\text { dys- } \\
\text { pnoea }\end{array}$ & $\begin{array}{l}\text { I30, } \\
\text { angina }\end{array}$ & & 9 & & 3 & & 9 & 8 & $\begin{array}{l}0 \\
0\end{array}$ \\
\hline
\end{tabular}

O, patent grant; $\bullet$, occluded graft; other abbreviations as in Table 2.

tion and 9 after. The average maximum pacing rate increased from 134 to I63 b.p.m. ( $P<0.00$ I). Angina was induced in all 32 patients before operation and in only ro after.

Average left ventricular end-diastolic pressure was 13.9 before and 14.2 after surgery. This difference is not significant. The average left ventricular dyskinesia score is unchanged at 0.9 and total coronary score did not change significantly (8.I to $8 \cdot 2$ ). Because the follow-up time in this subgroup is short, little information is available on the progression of lesions in the native circulation. It was noted, however, that approximately 25 per cent of the grafted vessels had become occluded proximal to the anastomosis.
Of the 52 grafts, $43(83 \%)$ were patent at the time of reinvestigation; $24(72 \%)$ of 33 patients had all grafts patent, $6(18 \%)$ had I patent and I occluded graft, and $3(9 \%)$ did not have a patent graft.

\section{Discussion}

The patients reported here represent approximately 40 per cent of all patients investigated for ischaemic heart disease during the period mentioned. The main indication for operation was the severity of angina and the way in which it limited any given patient's life. They in effect selected themselves in the majority by complaining of unacceptable symptoms in spite of treatment with nitroglycerin and beta-adrenergic blocking agents. 

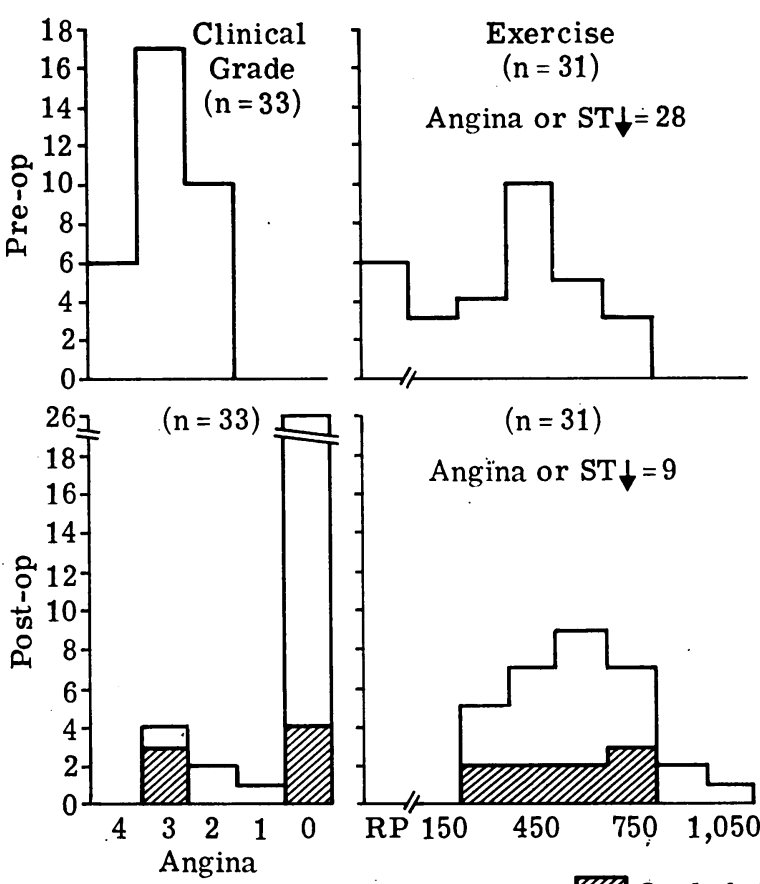

WOAccluded Graft

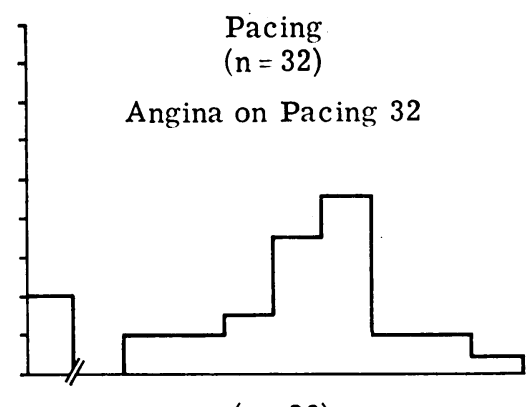

$(n=26)$

Angina on Pacing 10

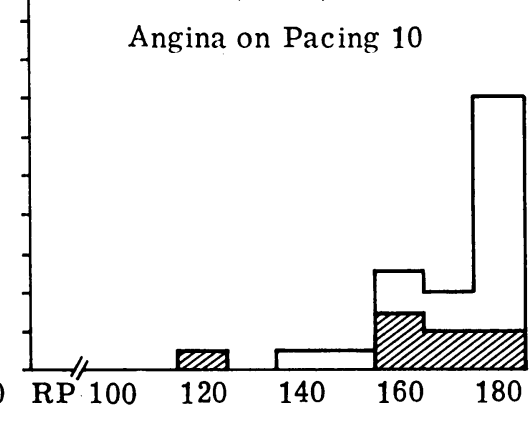

80

FIG. 2 The comparison of pre- and postoperative clinical grades and stress tests. $R P=$ Angina at rest; $S T \downarrow=S T$ segment depression of $>2 \mathrm{~mm}$.

Patients with severe left ventricular dysfunction were excluded, though the left ventriculogram was abnormal in 45 per cent. A few patients with severe diffuse coronary artery disease were also excluded at the beginning of the series but later bypass grafting with prior endarterectomy was used in some. The vein graft distribution and flows are similar to those previously reported (Johnson et al., 1970; Grondin et al., 1972; Danielson, Gau, and Davis, 1973). The operative mortality was 6.4 per cent which compared favourably with other series (Reul et al., 1972; Ross et al., 1972; Grondin et al., 1972; Manley and Johnson, 1972; Hammond and Poirier, 1973; Danielson et al., 1973), though 4 of the 7 deaths were in patients who had near normal left ventricular function preoperatively, and were caused by possibly avoidable complications, 3 deaths were caused by pulmonary embolism which did not occur after routine anticoagulation was used. Figures as low as 3 per cent have been achieved in the largest reported group (Favaloro, 1972; Sheldon et al., 1972). These figures have to be compared with the expected mortality without operation. The yearly figure in patients with angina pectoris is now recognized to be in the region of 3 to 4 per cent (Zukel et al., 1969; Kannel and Feinleib, 1972). This applies to a heterogeneous group of patients with all grades of disease. Recent publications have shown that the natural history is determined by the severity of the lesions. Patients with normal vessels have a lower mortality rate than the general population (Brushke, Proudfit, and Sones, 1973a) and survival decreases with increasing severity of disease (Freisinger, Page, and Ross, 1970; Bruhske, Proudfit, and Sones, 1973b). The overall one year mortality for patients with coronary artery lesions of at least 50 per cent was greater than Io per cent in a group with a distribution of disease very similar to ours (Brushke et al., 1973b). In our patients, using 75 per cent stenosis as the criterion for inclusion the operative mortality is lower than the one-year figure as reported for each of the 2 and 3 vessel disease groups. There have been no late deaths. Our patients also include 8 patients with major stenosis of the left main coronary artery (Table 2), all of whom survived, though this lesion is recognized to have a very high untreated mortality (Cohen et al., 1972; Brushke et al., 1973b). We did not define a subgroup with unstable angina, but there were 19 patients with recurrent pain at rest (grade 4; Table I) of whom 4 died. The operative mortality in the 90 remaining patients with stable 
angina of effort was 3.3 per cent. The overall figure did not therefore exceed that expected from available data on natural history.

Intraoperative studies have shown that occlusion of a newly anastomosed bypass graft adversely affects left ventricular function as judged by derivatives of left ventricular pressure (Bolooki et al., 1971). Early postoperative studies have shown varying effects on overall ventricular function (Marlon et al., I97I ; Mailhot, Sandler, and Harrison, I97I; Rees et al., 1971; Chatterjee et al., 1973) though contraction of hypokinetic areas has been shown to improve (Rees et al., I97I; Chatterjee et al., 1973). We saw no overall change in pre- and postoperative angiograms.

Of the 50 patients followed for more than 6 months, 36 are free of angina without drug therapy and a further I2 have improved. This excellent clinical result is similar to that obtained by others (Reul et al., I972; Adam et al., I972; Ross et al., 1972; Manley and Johnson, 1972; Friedberg, 1972). It has been suggested that the relief of pain may be due to perioperative myocardial infarction (Friedberg, 1972). Incidence of new $Q$ waves of up to 20 per cent has been reported (Ross et al., 1972). Our figure was 7 per cent of the survivors. A vectorcardiographic study (Friedberg et al., 1973) has suggested that the true incidence of new infarction is lower than that indicated by the scalar electrocardiogram.

In common with other comparable series our patients showed a significant improvement in exercise performance (Amsterdam et al., 1970; Manley et al., 1970; Lapin et al., 1973). The patients with abnormal preoperative left ventriculograms had lower exercise capability both before and after operation but showed similar improvement to those with normal left ventriculograms.

The patients who were reinvestigated also showed a significant clinical improvement and increase in exercise capability. The pacing stress test (Sowton et al., 1967), which provides a more objective assessment, confirmed these findings in both ours and Manley's patients (Manley et al., 1970). Of the 12 patients who were restudied because of chest pain, 7 had an occluded graft and all the patients who had no patent graft had postoperative angina. No patient was relieved of symptoms without a patent graft. Eighty-three per cent (43 of 52) of grafts were patent, a figure similar to that of other reported series (Sheldon et al., 1972; Flemma et al., 1972; Walker et al., 1972; Bourassa et al., 1972; Danielson et al., 1973). Approximately 25 per cent of grafted vessels became occluded proximally as has been previously observed (Malinow et al., 1973).

\section{Conclusions}

These initial results have shown that aorto-coronary bypass grafting is a relatively safe operation in our hands, with an operative mortality not exceeding the expected yearly mortality for an untreated group of patients. Both subjective and objective assessment have demonstrated considerable symptomatic improvement which is related to graft patency. We have been encouraged by these results to consider surgery earlier in the course of the disease in patients who are less limited by angina as we feel that it offers greater likelihood of complete relief of symptoms than medical treatment.

This work was supported by British Heart Foundation grants awarded to R. Balcon, A. F. Rickards, and R. K. Wilkinson.

\section{References}

Adam, M., Mitchel, B. F., Lambert, C. J., and Geisler, G. F. (1972). Long-term results with aorta-to-coronary artery bypass vein grafts. Annals of Thoracic Surgery, 14, I.

Amsterdam, E. A., Iben, A., Hurley, E. J., Zelis, R., Spann, J. F., and Mason, D. T. (1970). Physiological assessment of coronary vein bypass graft in refractory angina pectoris. Clinical demonstration of improved blood flow to ischaemic myocardium. Circulation, 41/42, Suppl. III, 129.

Bolooki, H., Rubinson, R. M., Michie, D. D., and Jude, J. R. (197I). Assessment of myocardial contractility after coronary bypass grafts. Fournal of Thoracic and Cardiovascular Surgery, 62, 543.

Bourassa, M. G., Lespérance, J., Campeau, L., and Simard, P. (1972). Factors influencing patency of aortocoronary vein grafts. Circulation, 45/46, Suppl. I, 79.

Brushke, A. V. G., Proudfit, W. L., and Sones, F. M. (1973a). Clinical course of patients with normal, and slightly or moderately abnormal coronary arteriograms. Circulation, 47, 936.

Brushke, A. V. G., Proudfit, W. L., and Sones, F. M. (1973b). Progress study of 590 consecutive nonsurgical cases of coronary disease followed 5-9 years. Arteriographic correlations. Circulation, 47, II 47.

Chatterjee, K., Swan, H. J. C., Parmley, W. W., Sustaita, H., Marcus, H. S., and Matloff, J. (1973). Influence of direct myocardial revascularization or left ventricular asynergy and function in patients with coronary heart disease. Circulation, 47, 276.

Cohen, M. V., Cohn, P. F., Herman, M. V., and Gorlin, R. (1972). Diagnosis and prognosis of main left coronary artery obstruction. Circulation, 45/46, Suppl. I, 57.

Danielson, G. K., Gau, G. T., and Davis, G. D. (1973). Early results of vein bypass grafts for coronary artery disease. Proceedings of the Staff Meetings of the Mayo Clinic, 48, 487.

Favaloro, R. (1972). Direct and indirect coronary surgery. Circulation, 46, II97.

Flemma, R. J., Johnson, W. D., Lepley, D., Tector, A. J., Walker, J., Gale, H., Beddingfield, G., and Manley, J. C. (1972). Late results of saphenous vein bypass grafting for myocardial revascularization. Annals of Thoracic Surgery, $14,232$.

Friedberg, C. K. (1972). Caution and coronary artery surgery. Circulation, 45, 727.

Friedberg, H. D., Zeft, H. J., Silberman, R. E., Tector, A. J., Jr., and Johnson, W. D. (1973). Myocardial infarction 
following coronary surgery: vectorcardiographic assessment (abstract). American fournal of Cardiology, 31, 132.

Friesinger, G. C., Page, E. E., and Ross, R. S. (1970). Prognostic significance of coronary arteriography. Transactions of the Association of American Physicians, 83, 78.

Grondin, C. M., Castonguay, Y. R., Lespérance, J., Bourassa, M. G., Campeau, L., and Grondin, P. (1972). Attrition rate of aorta-to-coronary artery saphenous vein grafts after one year. Annals of Thoracic Surgery, 14, 223.

Hammond, G. L., and Poirier, R. A. (I973). Early and late results of direct coronary reconstructive surgery for angina. Fournal of Thoracic and Cardiovascular Surgery, $65,127$.

Johnson, W. D., Flemma, R. J., Manley, J. C., and Lepley, D. (1970). The physiological parameters of ventricular function as affected by direct coronary surgery. Fournal of Thoracic and Cardiovascular Surgery, 60, 483.

Kannel, W. B., and Feinleib, M. (1972). Natural history of angina pectoris in the Framingham study. American fournal of Cardiology, 29, 154.

Lapin, E. S., Murray, J. A., Bruce, R. A., and Winterscheid, L. (1973). Changes in maximal exercise performance in the evaluation of saphenous vein bypass surgery. Circulation, 47, II64.

Mailhot, J., Sandler, H., and Harrison, D. C. (197I). Left ventricular function following coronary bypass surgery. Circulation, 43/44, Suppl. II, 196.

Malinow, M. R., Kremkau, E. L., Kloster, F. E., Bonchek, L. I., and Rösch, J. (1973). Occlusion of coronary arteries after vein bypass. Circulation, 47, I2I I.

Manley, J. C., and Johnson, W. D. (1972). Effects of surgery on angina (pre- and post-infarction) and myocardial function (failure). Circulation, 46, 1208.

Manley, J. C., Johnson, W. D., Flemma, R. J., and Lepley, D. (1970). Objective evaluation of the effects of direct myocardial revascularisation on ventricular function utilizing ergometer exercise testing (abstract). American Fournal of Cardiology, 26, 648.

Marlon, A. M., Adams, M. H., Enright, L. P., and Harrison, D. C. (197I). Effects of coronary artery bypass grafts on ventricular performance after chronic coronary artery occlusion. Circulation, 43/44, Suppl. II, 197.

Rees, G., Bristow, J. D., Kremkau, E. L., Green, G. S., Herr, R. H., Griswold, H. E., and Starr, A. (197r). Influence of aortocoronary bypass surgery on left ventricular performance. New England Fournal of Medicine, 284, I 116 .

Reul, E. J., Morris, G. C., Howell, J. F., Crawford, E. S., and Stelter, W. J. (1972). Current concepts in coronary artery surgery. Annals of Thoracic Surgery, 14, 243.

Ross, D. M., Sutton, R., Dow, J., Gonzalez-Lavin, L., Hendrix, G., Jefferson, K., McDonald, L., Petch, M., Smithen, C., and Sowton, E. (1972). Venous graft surgery in treatment of coronary heart disease. British Medical fournal, 2, 644 .

Sheldon, W. C., Rincon, G., Effler, D. B., Proudfit, W. L., and Sones, F. M. (1972). Vein graft surgery for coronary artery disease. Survival and angiographic results among the first 1000 patients. Circulation, 46, Suppl. II, I 10.

Sowton, G. E., Balcon, R., Cross, D., and Frick, M. H. (1967). Measurement of the angina threshold using atrial pacing. Cardiovascular Research, I, 301.

Walker, J. A., Friedberg, H. D., Flemma, R. J., and Johnson, W. D. (1972). Determinants of angiographic patency of aortocoronary vein bypass grafts. Circulation, 45/46, Suppl. I, 86.

Zukel, W. J., Cohen, B. M., Maltingly, T. W., and Hrubec, Z. (1969). Survival following first diagnosis of coronary heart disease. American Heart Fournal, 78, 159.

Requests for reprints to Dr. R. Balcon, The London Chest Hospital, Bonner Road, London E2 9JX. 